\title{
Dynamics of Macrostep Formation on Crystals: Recursive Differential Equations
}

\author{
J. P. v.d. Eerden and H. Müller-Krumbhaar
}

Research Institute for Materials Laboratory of Solid State Chemistry, Faculty of Science, University of Nijmegen, 6525 ED Nijmegen, The Netherlands and Institute für Festköperforschung, Kernforschungsanlage, D-5170 Jülich, Federal Republic of Germany.

\begin{abstract}
Dynamic coarsening of crystal surfaces by formation of macrosteps is explained by an improved model for impurity absorption. It is related to interface propagation in random media. Some unusual recursive equations are encountered and are briefly analyzed by functional iteration. The model is studied by various methods and exhibits some universal features. We find coarsening to proceed logarithmically with time in agreement with experiments.
\end{abstract}

During the growth of facetted crystals one frequently is faced with the problem, that the surface is not flat but covered with steps of dozens or hundreds of atomic units in height. This effect is generally undesired and also poorly understood. The usual reason for steps of about unit height are screw dislocations normal to the surface. There one end of a surface step is pinned, while the length of the step extends all over the surface. As the step advances during growth of the crystal, it winds up around the central screw dislocation, leading to a spiral step with almost constant distance between turns. Under constant growth conditions this spiraling step moves away from the center giving the impression of a spiral rotating with constant frequency. Observations on nearly perfect surfaces shown spirals with hundreds or thousands of tuns ("steps").

Sufficiently far away from the centre these spiral turns may be approximated as an equidistant step train, ignoring the curvature. The phenomenon under consideration [1-3] now is, that this step train generally has a tendency to form bunches, such that several unit steps move closer together leaving large terraces between these bunches. As no static forces (like elastic stress) are known to produce this effect, it is assumed that this is an intrinsically dynamic phenomenon.

We will describe a model that produces this feature in good agreement with experiments. In addition we encounter some unusual recursive differential equations, which will be discussed for their own interest at the end.

In addition to this concrete application there is an analogy to coarsening in "spinodal decomposition" if one regards the flat terraces as parts of phase $A$ and the macrosteps as parts of phase B. Furthermore, there is a close relationship to the propagation of interfaces in random media, as briefly discussed later. .

Our basic assumption is that a small concentration of impurities adsorbed on the "terraces" between surface steps may play an essential role in many coarsening phenomena, as supported by experiments [1-3]. Various models were previously defined starting from very similar ingredients, [4-7] but they lack some important cooperative effects. Some other models rely on rather arbitrary assumptions.
We assume that the substrate material, forming the growing crystal, impinges from the vapor or solution onto the terraces between steps, quickly reaches quasiequilibrium (in relation with the vapor), and finally crystallizes into a nearby step by surface diffusion. This advances the step. Impurities also impinge onto the terraces, where they remain immobile, also reaching quasiequilibrium exponentially with time [7], but on a slower scale than the substrate material [1]. These random impurities hamper the rate of advancement of steps to an extent that depends on their concentration immediately in front of a step [4-6]. As the step advances impurities are incorporated, leaving an essentially "clean" surface directly behind the step.

A particular position on the surface thus undergoes oscillations in impurity concentration as steps are passing by. Consequently, the velocity $v_{n}$ of step $n$ is basically a function of the time $\tau_{n}$ elapsed since the step ahead has passed the same position $y_{n}(t)$. More precisely, this is formulated as

$y_{n}(t)=y_{n+1}\left(t-\tau_{n}\right)$.

$v_{N}(t)=V\left(\tau_{n}(t)\right)$.

where $n$ denotes a specific step, $n+1$ the step in front of it. Because of the exponential saturation of impurities with time, $V(\tau)$ asymptotically goes as $V_{0}+\delta V \times \exp (-\lambda \tau)$ for large $\tau$. For small exposure times $\tau_{n}$ the velocity $v_{n}$ decreases with decreasing $\tau_{n}$ because of the lack of a supply of substrate material between densely spaced steps. The step velocity $V(\tau)$, therefore, first increases with $\tau$, goes through a maximum at $\tau_{m}$, then decreases towards $V_{0}$. This completes the basic one-dimensional model.

Our model turns out to be closer to experiments in formulation and results than previous theories [4-7] which have not considered the collective effects produced by this natural "exposure time" formulation, and which leads to quite different results [6].

Despite its simplicity the model (1) shows surprising features. Dynamics follow by taking the time derivative of (1a), using (1b) with $\mathrm{d} y_{n}(t) / \mathrm{d} t=v_{n}=V\left(\tau_{n}\right)$ :

$\frac{\mathrm{d} \tau_{n}(t)}{\mathrm{d} t}=1-\frac{V\left(\tau_{n}(t)\right)}{V\left(\tau_{n+1}\left(t-\tau_{n}\right)\right)}$.

This is an unusual differential-difference equation: It is recursive, i.e., the set of functions $\tau_{n}(t)$ to be found appear in their own argument! It has only a first time derivative but, in order to formulate initial conditions, one has to know the solution [8]. For long times, however, the distances between steps vary slowly in comparison with the typical exposure times, so that we may consistently replace $\tau_{n} V_{n+1}$ by $\mathrm{d}_{n}=y_{n+1}(t)-y_{n}(t)$. This then gives an approximate set of differential equations 
for $\mathrm{d}_{n}(t)$ [9], tractable by computer or by other methods discussed below.

Even for the full set (2) a linear stability analysis is readily performed. A constant velocity $V_{a v}\left(V_{m}>V_{a v}>V_{0}\right)$ for all steps corresponds to two exposure times $\tau<\tau_{m}$ and $\tau_{b}>\tau_{m}$. Making a small perturbation $(\sim \varepsilon)$ around $\tau_{b}, \tau_{n}(t)=\tau_{b}+$ $\varepsilon \exp (\mathrm{i} k n+\Omega t)$, one arrives at

$\Omega=\left(Q / \tau_{b}\right)\left[\exp \left(\mathrm{i} k-\Omega \tau_{b}\right)-1\right]$,

with $Q=\mathrm{d}[\ln V(\tau)] / \mathrm{d}(\ln \tau)$. Here $k$ is the wave number of the perturbation. The system is unstable for $\tau_{b}>\tau_{m}$, meaning $Q<0$, the maximal instability occuring at $k=\pi$. If we start with a nearly equidistant step train with $\tau \simeq \tau_{b}$, this means pairwise coalescence of steps. In other words, this coarsening process starts if the typical exposure times initially are around or larger than $\tau_{m}$. (The singularity produced by the kinematic wave theory [5] for a continuum model depends sensitively on the initial distribution of steps, as already noticed by Frank, and hence differs substantially from the present result.) Note further that $V_{a v}=$ const still allows for an enormous multiplicity of (dynamically) stationary states, as only the two exposure times then are fixed but not the actual distribution of steps. As can easily be deduced, the pairwise coalescence continues to hold also for macrosteps, i.e., bunches of steps (within which there is the short exposure time $\tau_{\alpha}$ ). This step doubling lives on the discrete nature of (2) and is lost in continuum approximations $[5,6]$.

In order to study the long-time behavior of this process we consider the distribution function $S(\tau, t)$ for exposure times $\tau$ at observation time $\tau$. Initially it may start as a unimodal function at $t=0$. It is then quickly split into a bimodal structure, peaked around two values $\tau_{\alpha}(t)<\tau_{m}$ and $\tau_{\mathrm{b}}(t)>$ $\tau_{m}$. Here $\tau_{\alpha}$ corresponds to bunching of steps into macrosteps, while $\tau_{b}$ corresponds to the large terraces between macrosteps. According to the stability analysis (3) the distribution $S(\tau, t)$ is stable (contracting) around $\tau_{\alpha}$. This peak, therefore, serves as a "sink" for exposure times $\tau_{n}$. Alternatively, the peak around $\tau_{b}$ represents the "source" and is decreasing during the coarsening process. The latter peak thus dominates the systems dynamics and accordingly can be viewed as a unimodal distribution. More pictorially, the dynamics of a macrostep is governed by the leading step of the bunch. The peak around $\tau_{\alpha}$ then merely serves to maintain global conservation of steps.

This conservation law locally can be written as

$\partial_{t} S(\tau, t)=-\partial_{\tau}[u(\tau, t) S(\tau, t)]$,

where $u$ is the (drift) rate of change of $\tau$ values. Defining step distances $\mathrm{d}_{n}=\tau_{n} V\left(\tau_{n+1}\right)$ as above, we obtain

$V\left(\tau_{n+1}\right)-V\left(\tau_{n}\right)=\left(\partial_{t} \tau_{n}\right) V\left(\tau_{n+1}\right)+\tau_{n} \partial_{t} V\left(\tau_{n+1}(t)\right)$.

We define a mean-field approximation by

$$
\begin{aligned}
V\left(\tau_{n+1}\right) \rightarrow\langle V(t)\rangle & \equiv W(t) \\
& =\int \mathrm{d} \tau V(\tau) S(\tau, t) / \int \mathrm{s} \tau S(\tau, t)
\end{aligned}
$$

stating that the velocity of step $n+1$ in front of step $n$ be replaced by the average velocity $W(t)$ over all steps. Inserting (6) into (5) and identifying now $\partial_{t} \tau_{n}(t)=u(\tau, t)$, we obtain

$u(\tau, t)=1-V(\tau) / W(t)-\tau \partial_{t} W(t) / W(t)$.

The set of eqs. (4), (6) and (7) defines the functional meanfield equation for the dynamics of the distribution function

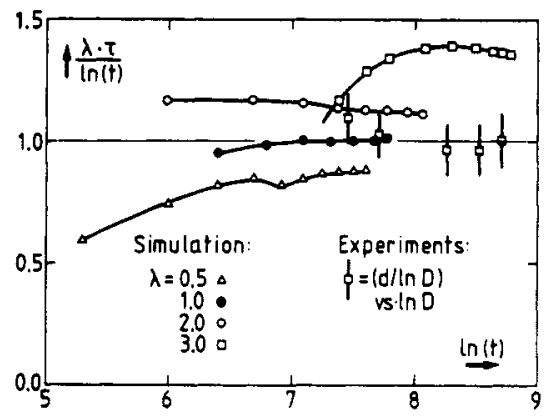

Fig. 1. Comparison of the prediced scale relation (12) with computer simulations (Ref. [9]) and experiments (Ref. [1]). Since no experimental values for time scales $\tau$ were available we have taken experimental data for step spacing $d$ and distance $D$ from the spiral center. For long times all data converge towards the scaling relation.

$S(\tau, t)$. To be precise, it only describes the dynamics of the peak near $\tau>\tau_{m}$. But for long times we shall observe $0<$ $\tau_{\alpha}<\tau_{m} \ll \tau_{b}$, and so we may assume (4) to hold in the full range $\tau>0$. The leakage out of $S(\tau, t$ at $\tau=0$ then contributes to the peak at $\tau_{\alpha}$ (global conservation) which then need not be considered further.

The asymptotical behavior of the peak around $\tau_{b}=\tau_{0}(t)$ can be qualitatively understood by some simple arguments. At a certain time $t$ assume that the step velocities scatter around their average value by an amount $\Delta v$ on average. The average step-distance being

$\delta=V\left(\tau_{0}\right) \cdot \tau_{0}$

may be assumed to increase as

$\frac{\mathrm{d}}{\mathrm{d} t} \delta=\frac{1}{T} \delta$

with $T$ being the typical coalescence time

$T=\delta / \Delta V=\frac{\tau_{0}}{\Delta \tau_{0}}\left|\frac{V\left(\tau_{0}\right)}{V^{\prime}\left(\tau_{0}\right)}\right|$.

Here $\Delta \tau_{0}$ is the width of the peak near $\tau_{0}, V^{\prime}\left(\tau_{0}\right)$ means derivative with respect to the argument. With the above definition of $\delta$, eqs. $(9,10)$ are readily transformed into

$\delta_{t} \tau_{0}(t)=\Delta \tau_{0}\left|\frac{V^{\prime}\left(\tau_{0}\right)}{V\left(\tau_{0}\right)}\right|$

to leading order for large $\tau_{0}$. Here we have assumed that $V(\tau)$ behaves as defined after eq. (1). (This reasoning is only valid, if $\Delta \tau_{0} / \tau_{0} \rightarrow 0$ for long times. A better derivation is indicated in Ref. [9]). From (11) follows immediately the asymptotic result:

$\tau_{0} \lambda=\ln (t)$

Because of this logarithm, the single experimental constant $\lambda$ determines the results. It is the ratio of impurity atoms impinging on the surface per area and time to the equilibrium concentration at a flat surface. This scaling result is essentially a consequence of the exponential decay of $V(\tau)$.

A comparison [Fig. (1)] with direct computer simulations [9] shows agreement with the predicted asymptotic scaling (9) and with an analysis [9] of recent experimental data [1]. For small values of $\lambda$ the convergence of the numerical data towards (9) of course is faster than for large ones. Other parameters like $V_{0}, \delta V, \tau_{m}$, etc., affect the short-time behavior only. The agreement with experimental results [1] is also 
obvious. The plot shows $d$, the spacing between macrosteps, and $D$, the distance from the spiral center, both in arbitrary units. Previous attempts [6] suggesting a $t^{1 / 2}$ law on a more phenomenological basis, not explicitly considering impurities, are inconsistent with these experiments. However, we do not claim that the formation of macrosteps is always a consequence of the mechanism described here. The combined action of diffusion and kinetic coefficients was taken into account in an averaged way only, but in any case impurities even at small concentrations seem to play a central role in the kinetics of step movement. Furthermore, we have ignored fluctuations along the steps. As in the general problem of a interface moving in a random medium [10], they presumably are significant in crystals with small anisotropy of the step free energy.

Returning from the picture of an (almost parallel step train to the original picture of a spiral centered at a screw dislocation, this step bunching leads to larger and larger terraces between macrosteps as one goes from the center on outwards. The overall structure thus appears to have a "fractal" property. Denoting the distance of a macrostep from the center by $r$, one may define a fractal dimension $D$ by

$\lim _{R \rightarrow \infty} R^{D}=\lim _{R \rightarrow \infty} \int_{\epsilon}^{R} \mathrm{~d} r r \varrho(r)$

where $\varrho(r) \sim 1 / \ln (r)$ is the density of macrosteps in radial direction, according to (12). $\in$ is some irrelevant lower cutoff. The result is

$D=2-\ln (\ln R) / \ln R$

which asymptotically converges to 2 , but on an extremely slow scale!

In the remaining part of this article we will shortly look at some unusual properties of recursive differential equatons as suggested from eq. (2).

For simplicity we will ignore the physical origin and just ask, how to solve a time-delayed differential equation, where the time-delay is the unknown function itself:

$\partial_{t} x(t)=x(t-x(t))$.

(Further examples are discussed in Ref. [8]). Let us look for a solution with $x(t)>0$ at some $t, x(t)$ being continuous and defined for all $t \in(-\infty, \infty)$. Assuming $x(t) \rightarrow+0$ for $t \rightarrow-\infty$, we may ignore $x(t)$ in the argument, such that eq. (15) seems to approach an exponential function for $t \rightarrow-\infty$.

A simple exponential function has the trivial property, that a shift of the argument can be identically compensated by a multiplication of the prefactor. This reminds us of a renormalization procedure, where a change of scale (here: shift) is compensated by a change of coupling constants (here: prefactor). Applying this idea to our eq. (15), we define the renormalization $f(\mathrm{t})$ by

$x(t-x(t))=f(t) \cdot x(t)$

Inserting (16) into (15) we obtain

$x(t)=\exp \left\{-\int_{t}^{0} \mathrm{~d} t^{\prime} f\left(t^{\prime}\right)\right\}$

and

$f(t)=\exp \left\{-\int_{t-x(t)}^{t} \mathrm{~d} t^{\prime} f\left(t^{\prime}\right)\right\}$

for $t \leqslant 0$. It turns out, that eqs $(17 \mathrm{a}, \mathrm{b})$ already form a converging scheme for functional iteration (to be performed by computer). Insert some $f(t)$ into (17a), calculate $x(t)$. Then insert those trial-functions into $(17 \mathrm{~b})$, to obtain a new trialfunction $f(t)$. The resulting (numerically obtained) function [8] looks for $t \rightarrow-\infty$ like an exponential function, has at $t=0$ the value $x(0)=1$ and $\partial_{t} x(t=0)=0.5434 \ldots$ and goes for $t \rightarrow \infty$ towards $t$ as $x(t)-t \sim \exp (-\dot{x}(0) \cdot t)$. Here we have made use of the translational invariance $t \rightarrow$ $t+$ const. to set $x(0)=1$. Note that direct numerical forward-integration instead of using (17) is not possible, since one is not free to chose "initial" conditions.

Concrete applications of such equations can possibly be made in population dynamics, but this is beyond the scope of the present article.

\section{References}

1. van Enckevort, W. P. J., Prog. Cryst. Growth Charact. 9, 1 (1984).

2. Bloem, J. and Giling, L. J., Curr. Top. Mater. Sci. 1, 147 (1978).

3. Tsukamoto, K., J. Cryst. Growth 61, 199 (1983).

4. Cabrera, N. and Vermilyea, D. A., in Growth and Perfection of Crystảs, (Edited by R. Doremus, B. Roberts, and D. Turnbull), p. 393. Wiley, New York (1958).

5. Frank, F. C., in Ref. [4], p. 411.

6. Chernov, A. A., Usp. Fiz. Nauk, 73, 277 (1961) [Sov. Phys. Usp. 4, 116 (1961)].

7. Chernov, A. A., Parvov, V. F., Kliya, M. O., Kostomer, D. V. and Kuznetsov, I. G., Kristallografiya 1125, 640 (1981) [Sov. Phys. Crystallogr. 26, 640 (1981)].

8. Drastically simplified ordinary recursive equations were studied in Müller-Krumbhaar, H. and Eerden, J. P. v.d., Z. Physik B (to appear). There are obvious relations between such equations and an equation studied in Reich, L., in Iteration Theory and Its Functional Equations, edited by Liedl, R., Reich, L. and Targonski, Gy., Springer-Verlag, Heidelberg (1985), p. 135; Beyes, W.a. and Channell, P. J., ibid., p. 7.

9. Eerden, J. P. vd. and Müller-Krumbhaar, H., Phys. Rev. Lett. 57, 2431 (1986).

10. Bruinsma, R. and Aeppli, G., Phys. Rev. Lett. 52, 1547 (1984). 